\title{
NOVEL GENES AND HORMONES IN SALIVARY GLANDS: FROM THE GENE FOR THE SUBMANDIBULAR RAT 1 PROTEIN (SMR1) PRECURSOR TO RECEPTOR SITES FOR SMR1 MATURE PEPTIDES
}

\author{
Catherine Rougeot, Isabelle Rosinski-Chupin, and Fransois Rougeon \\ Unit of Genetics and Biochemistry of Development, National Center of Scientific Research, and \\ Department of Immunology, Institute Pasteur, Paris, France
}

\section{SUMMARY}

- $\quad$ Siibmandibular rat 1 protein (SMR1) preprohormone and its maturation peptides constitute the novel cha racterized submandibular gland (SMG)-specific factors of the cervical sympathetic trunk (CST)-SMG (CST-SMG) axis. As is generally observed for major polypeptide hormones of the endocrine system, SMR1 peptides, including SMR1imdecapeptide, -hexapeptide and-pentapeptide, are selec tively matured from the precursor by cleavage at pairs of basic residues, and differentially accumulated and locally as well as systemically released under multifactorial $n$ euro endocrine control. In turn, the final SMRl mature pentapeptide, at hormonal circulating concentrations, is selectively taken up by peripheral targets through specific binding sites. Localiza tion of the target cells suggests that the SMRl-derived pen tapeptide might be involved in modulating mineral ion bal ance in vivo. Furthermore, associated with male rat specific behavioral characteristics, one can propose that the androgen-regulated SMRl-pentapeptide is a SMG hormonal factor, which under stressful circumstances, is acutely secreted to counterregulate the mineral homeostatic responses to stress.

Received for publication 15 May 1998 and accepted 20 November 1998.

Corespondence and reprint requests to Dr Catherine Rougeot. Unit of Genetics and Biochemistry of Development. Department of Immunology, Institute Pasteur. 25 rue du DrRoux. F-75724 Paris Cedex 15. France. Tel: 33 (01) 4061 3445, Fax: 33 (01) 4061 3440. E-mail: crougeotSjpasteur.fr
The gene VCSA1, which encodes SMRl, belongs to a new muItigene family, essentially expressed in the salivary glands of mammals. This family, whose several members also display putative sites of processing by convertases, has an unusual evolution characterized by multiple gene duplications and an accelerated divergence of coding sequences. (Biomed Rev 1998; 9: 17-32)

\section{INTRODUCTION}

- When studying submandibular glands (SMG) and their products, two observations are particularly puzzling: (i) these glands secrete in large quantities hormones, growth fac tors and enzymes mostly known for their systemic functions $(1,2)$, and (ii) important species differences and frequent sexual dimorphism, especially in rodents, are observed at the histological and biochemical levels. In particular, the proteins synthe sized by the SMG are quite different both quantitatively and qu alitatively from one species to another. The diversification of the salivary proteins according to species and the existence of sexual dimorphism, led us and others, to propose that some sali vary polypeptides might be involved in the establishment of species specific characteristics in relation with male mecha nisms by which the corresponding genes have been recruited to fulfill these specific functions include changes in the regula tion of gene expression, and/or appearance of salivary gene variants with modified functions by gene duplication followed by sequence divergence. For instance, renin is an enzyme pro duced by the juxtaglomerular cells of the kidney which is 
involved in the regulation of blood pressure and hydromineral homeostasis. In the male mouse, an important extrarenal source for renin synthesis is the SMG (3). Renin of salivaty origin is not glycosylated and differs from renal renin by its lower stability (4). Salivary renin is secreted both into the saliva and the blood, particularly during the aggressive behaviors of male mice (5). However, no effect of this secretion on peripheral blood pressure has yet been demonstrated. From the genetic point of view, renin synthesis in the SMG is linked to a gene duplication. This gene duplication is only found in the mouse which is the only species known to synthesize renin in the SMG (6-9). In the rat SMG, a number of genes are expressed at a higher level in males than in females, e.g. the genes encoding kallikreins, epidermal growth factor (EOF), transforming growth factor-beta (TGF-p),andsubmandibularrat 1 and2proteins(SMR1,SMR2), respectively.

\section{CHARACTERIZATION OF THE GENE ENCODING A NEW POLYPEPTIDE HORMONE PRECURSOR NAMED SMR1}

\section{- $\quad$ Structure of the VCSA1 gene encoding SMR1}

When the in vitro translation products from RNA obtained from SMG of male and female rats are compared, some polypeptides appear differentially expressed according to the sex (10). SMR1 was firstly described as one of these polypeptides whose expression is higher in males than in females. Using a differential screening strategy, we cloned the corresponding cDNA from a SMG cDNA library (10). Analysis of the SMR1 cDNA sequence led us to postulate that SMR1 might be a new preprohonnone: the sequence of SMR1 contains signals for secretion and processing by convertases. The sequence and structure of the gene encoding SMR1, also called the VCSA1 gene, have been elucidated(1 1,12).The VCSA1 gene is on rat chromosome 14/ p21 -22 (13). Different mRNAs, generated by differential maturation of the primary transcripts, have been characterized in the rat SMG (12). Four of them only differ by the length of their untranslated regions (3' UTR) and all encode SMR1. The most abundant and first described transcript is about $700 \mathrm{~b}$ long. It contains 438 nucleotides (nt) ORF encoding the SMR1 poly-peptide, together with 86 and 142 nt 5' and 3' UTR, respectively. Sequence comparison between cDNA and genomic DNA reveals the presence of two introns. In the mature mRNA, exon 1 corresponds to most of the 5'UTR, exon 2 encodes most of the signal peptide, and the third exon which is unusually long (more than $500 \mathrm{nt}$ ) encodes the last 4 amino acids of the signal peptide, the whole mature protein and the 3UTR. Interestingly, the structure of the VCSA1 gene is reminiscent of the structure of several genes encoding prohormones, such as the genes for preprothyrotropin-releasing hormone, preproenkephalins or preproopiomelanocortin (11). Whether this structure reflects the existence of a common ancestor, or convergent evolution, is at present not known.

\section{$\bullet$}

\section{The VCSA1 gene encoding SMR1 is a member of a} new multigene family, the VCS family

Probing genomic DNA blots with SMR1 cDNA showed that a number of related sequences are found in rat genome $(10,14)$. Subsequent cloning and characterization of a number of genes related to SMR1 gene in the rat, but also in the mouse and in the human, confirmed that it belongs to a multigene family. Sequencing of some of these related genes revealed the presence of a "hypervariable sequence" covering a large part of the coding sequence (about 150-200 nt), where sequence similarity sharply drops. For this reason, this family has been named variable coding sequence (VCS) family, and the SMR1 gene was also called VCSA1 gene. As a result of this variation, the protein sequences also differ notably in this region (Fig. 1). Although located outside the hypervariable sequence, the C-terminus of the proteins is also variable in length, which introduces an additional level of variability between VCS gene products.

Whereas the VCS genes encode proteins which are very different in sequence and therefore probably in function, they nevertheless share a number of properties which enable one to group them into a family. Indeed, the different genes characterized to date, generally present $(i)$ a common structure resembling that of VCS A1, (ii) sequence similarities which are mainly observed in the first and second exons, as well as in the beginning and the 3 ' end of the third exon, and $(\mathrm{Hi})$ at the amino acid level, a relatively good conservation of the 35 first amino acids of the VCS gene products, with the first 20 amino acids corresponding to the signal peptide, i.e. the VCS gene products are most likely secreted. The RXYR sequence, generally RGPR, which is a good recognition site for convertases $(15,16)$, is also conserved in the N-terminus of VCS gene encoded proteins. Therefore, most of the VCS gene products might be proteolyti-cally processed at this site. A common chromosomal localization in the rat all the VCS genes characterized so far have been mapped in chromosome 14/p21 -22 (13). Due to conservation of syntenic groups between rat, mouse and human, the VCS genes are expected to map on mouse chromosome 5 and human chromosome 4 . Indeed this localization has been confirmed for one of the mouse VCS genes (Senorale $e t a l$, submitted for publication) and one of the human VCS genes. The main characteristics of some of the known VCS genes are summarized in Table 1.

The VCSA genes, VCSA1 (SMR1 gene), VCSA2 and VCSA3, form a subgroup of closely related genes in the rat and encode SMR1 and SMR1-related polypeptides (17). These polypeptides, which contain potential recognition sites for proteolytic enzymes, can all be considered as potential preprohormones whose expression is spatiotemporally regulated. While VCSA 1 is mostly expressed in the SMG of sexually mature male rats, VCS A2 is expressed in young ( $0-18$ days after birth) rat glands 


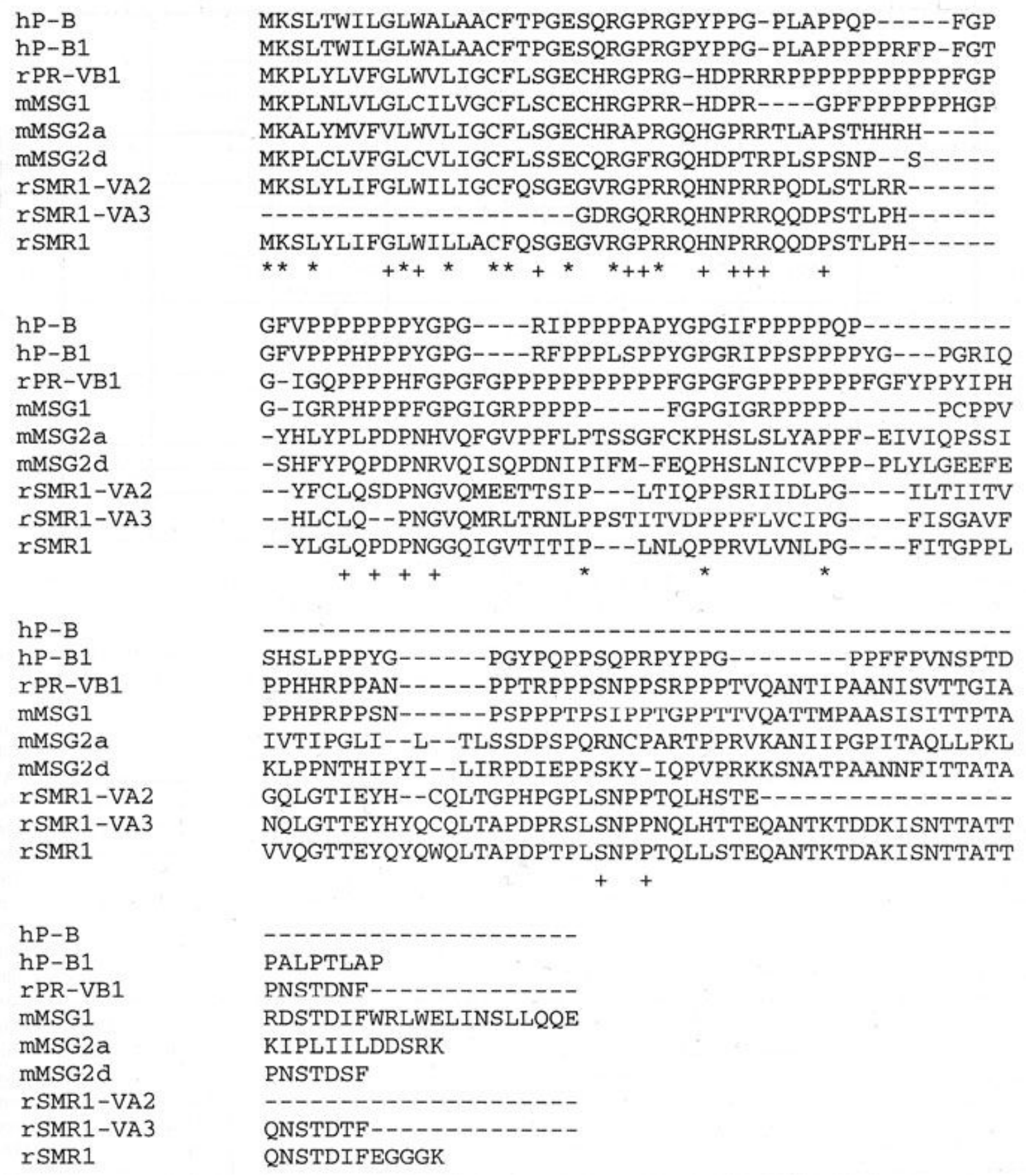

Figure 1. Sequence comparison of VCS gene proteins. * amino acid conservation in all sequences; + , a conservation in at least 6 among the 9 sequences. 
Table 1. Main characteristics of the VCS genes known in rat, mouse and human

\begin{tabular}{|c|c|c|c|c|c|c|c|}
\hline & \multirow[t]{2}{*}{ Gene } & \multicolumn{3}{|c|}{ protein } & \multicolumn{2}{|c|}{ Expression } & \multirow[t]{2}{*}{ ref } \\
\hline & & Name & $\begin{array}{l}\text { Precursor of } \\
\text { aLMW } \\
\text { peptide }\end{array}$ & $\begin{array}{l}\text { proline-rich } \\
\text { unit }\end{array}$ & Tissue & \begin{tabular}{|l|} 
androgen \\
regulation
\end{tabular} & \\
\hline \multirow[t]{4}{*}{ Rat } & VCSAI & SMR1 & Yes & No & \begin{tabular}{|l|}
$\begin{array}{l}\text { SMG+++ } \\
\text { prostate }\end{array}$ \\
\end{tabular} & +++ & 10 \\
\hline & VCSA2 & SMR1-VA2 & potential & No & SMG & - & 17 \\
\hline & VCSA3 & $\begin{array}{l}\text { SMR1- } \\
\text { VA3? }\end{array}$ & potential & No & 7 & 7 & 17 \\
\hline & $V C S B 1$ & PR-VB1 & potential & Yes & \begin{tabular}{|l|} 
SMG? \\
Parotid+++
\end{tabular} & - & 14 \\
\hline \multirow[t]{2}{*}{ Mouse } & Vcsl & MSG1 & potential & Yes & $\begin{array}{l}\text { parotid+++ } \\
\text { SMG? skin? }\end{array}$ & & 19 \\
\hline & Vcs2 & $\begin{array}{l}\text { MSG2a } \\
\text { MSG2d } \\
\text { MSG2g }\end{array}$ & \begin{tabular}{|l|} 
potential \\
potential \\
potential
\end{tabular} & No & SMG & + & 18 \\
\hline \multirow[t]{3}{*}{ Human } & $P B$ & P-B & No & Yes & SMG & - & 21 \\
\hline & $F B I$ & P-B1 & No & Yes & 7 & 7 & 20 \\
\hline & $B P L P$, & BPLP & No & Yes & $\begin{array}{l}\text { lacrimal } \\
\text { gland }\end{array}$ & 7 & 24 \\
\hline
\end{tabular}

(Rosinski-Chupin and Courty, unpublished data), and no site of expression for VCSA3 has been characterized to date. Sequence analysis of these genes reveals that they evolved after recent duplications of an ancestor (17).

VCS2 is the mouse gene most related to the VCSA subgroup $(18,19)$. It is expressed in mouse SMG in response to androgens, like VCSA 1, but at a lower level. Its structure is relatively unusual for a member of the VCS family since it is composed of 6 exons, due to the juxtaposition of three VCS sequences following events of gene fusion. Five mRNAs resulting from alternative splicing of the same primary messenger were characterized. Three of the encoded proteins have a structure similar to SMR1 and might be processed to release low molecular weight pep-tides.

The rat VCSB1, the mouse VCS 1, the human H-PB and H-PBI genes encode polypeptides containing repetitions of a prolinerich sequence, GPGXXPPPP (14,19-22). These polypeptides also contain potential sites for maturation enzymes. The mouse and rat genes are expressed in the acinar cells of parotids, while H-PB is expressed in the human SMG. The presence of potential sites for bacterial collagenases lead to proposition that these proteins might have a role in buccal protection by inhibiting collagenases. In addition, arole in tooth mineralization has been also proposed after the discovery of a P-B-like peptide as a tooth enamelin (23). Based on nucleotide similarities in the 5'UTR region and the beginning of the coding sequence, a fifth gene, encoding a lacrimal proline-rich protein in humans also seems to belong to this group of VCS genes (24).

\section{- Evolutionary events leading to VCSA1 gene expres sion in rat submandibular glands}

VCSA1 (SMR1) gene belongs to a family whose major site of expression is the salivary glands. Sequence comparisons with related genes in the same or different species reveal that VCSA 1 is a relatively new gene, specific for the Rattus genus, whose sequence has diverged from other VCS sequences after gene duplication. In fact, VCSA1 coding sequence can be split into several regions apparently evolving at different rates. The hypervariable region is hardly conserved in other VCS genes and would appear to have evolved at an accelerated rate. Studying the VCSA subgroup of genes in the Rattus genus, lead to the conclusion that sequence divergence in this region is due to the presence of multiple insertions, deletions or mutations. Interestingly, most of these mutations are not silent and lead to amino acid substitutions, suggesting that evolution of this region might be driven by mechanisms promoting sequence diversification (17). Contrasting with the hypervariable region, the first $\mathrm{N}$-terminal amino acids which include the signal peptide and the first potential proteolytic site are well conserved between the human, mouse and rat genes. The adjacent sequence which corresponds to the polypeptide hormone is found more or less conserved and is followed by a second putative proteolytic site in all proteins except in the human prolinerich 
proteins. Whether this sequence has been recently gained during evolution, or has been lost in human proline-rich proteins, is not known and a more extensive characterization of VCS genes in other species is necessary to answer this question.

In conclusion, this dual evolution might signify that at least two different functions are carried by the VCS gene encoded proteins: one function, linked to the capacity to generate low molecular weight peptides, is relatively common to the family, and the other function carried by the hypervariable peptidic region would be more polypeptide-specific.

\section{MATURATION PEPTIDES OF SMR1 PRECURSOR}

\section{- $\quad$ SMR1 maturation polypeptides}

Hormonal precursors of the endocrine system generate biological active peptides by selective proteolytic cleavages at paired and single basic amino acids $(26,27)$. The SMR1 protein struc- ture displays features common to these polypeptide hormone precursors: it contains an $\mathrm{N}$-terminal secretory signal sequence of hydrophobic amino acids and sites for a limited and controlled endoproteolytic process. On the one hand, two potential sites of paired arginine residues are present in the $\mathrm{N}$-terminus of the sequence R27-R28 and R33-R34 (Fig. 2) and have the environmental characteristics required to direct cleavage by the paired basic amino acid maturases, such as furin which is widely distributed within the rodent SMG (28). Thus, the two dibasic sites encompass, in a hydrophilic region, a tetrapeptide (QHNP) and basic amino acid occupies the $\mathrm{P} 4$ position relative to each cleavage site (R/H-X-Y-R-R). On the other hand, a single basic amino acid is present in the median part of the SMR1 sequence R 70 (Fig. 2). Single and pairs of basic amino acids are essential processing sites for the generation of biologically active pep-tides. However, their selection is limited and dictated by the nature and activity of converting enzyme as well as by the primary, secondary and tertiary structure of the precursor and by the tissue environment in which it is produced $(26,27,29,30)$.

\title{
SMR1 preproprotein \\ and \\ SMR1 maturation peptides
}

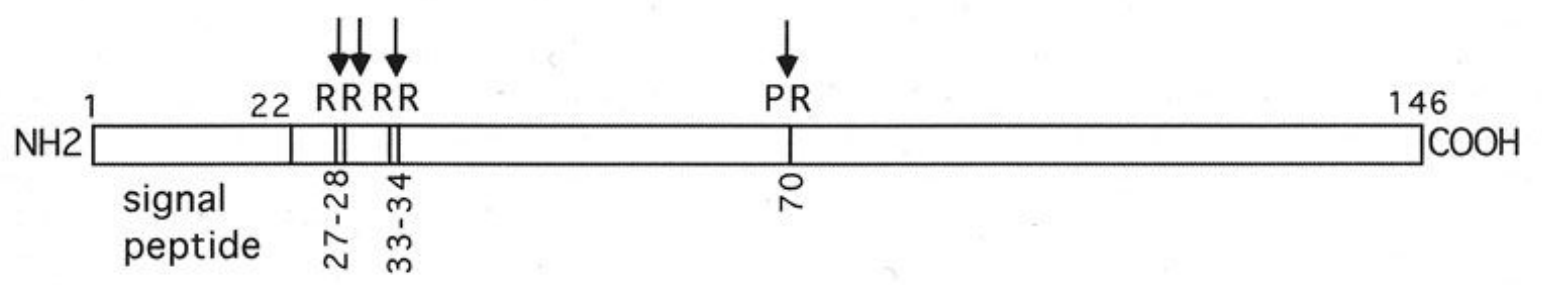

\author{
Val-Arg-Gly-Pro-Arg-Arg-Gln-His-Asn-Pro-Arg SMR1-Undecapeptide \\ Arg-GIn-His-Asn-Pro-Arg SMR1-Hexapeptide \\ Glp-His-Asn-Pro-Arg SMR1-Pentapeptide \\ Gln-His-Asn-Pro-Arg
}

Figure 2. Schematic diagram ofSMRI preprotein structure and of its derived peptides which are maturated from precursor by selective cleavages at dibasic Arg-Arg sites. The processing mono- and dibasic sites are denoted by arrows. The single and pairs of basic residues are given by single-letter amino-acid symbols. Three structurally related peptides have been characterized from the SMG of adult male rats. All are generated from SMRI precursor by selective cleavages at both Arg-Arg bonds. 
In vivo, SMR1 protein is selectively processed at pairs of basic amino acid sites in a tissue- and sex- specific manner to give rise to mature peptide products, in a manner similar to the maturation pathway of polypeptide hormone precursors (31). Three structurally related peptides have been isolated and characterized from the SMG and prostate of adult male rats. Theundecapepti-de VRGPRRQHNPR is generated from SMR1 by selective en-doproteolysis at the Arg33-Arg34 bond and at the signal peptide cleavage site. The hexapeptide RQHNPR and the pen-tapeptide QHNPR are generated by selective cleavages at both $\operatorname{Arg} 27-\operatorname{Arg} 28$ and $\operatorname{Arg} 37-\operatorname{Arg} 38$ bonds occurring either between or following pairs of basic residues. The final processed peptide named SMR1 -pentapeptide appears in two forms, one with the N-terminal glutamine and other with the N-terminal py-roglutamate (Fig.2) (31). Furthermore, as revealed by in vitro metabolic experiments employing radiolabeled peptide added to various biological fluids and tissue extracts, the SMR1 undeca-peptide is the direct precursor of the SMR1-hexapeptide and SMR 1 -pentapeptide in the rat SMG and its secretions into saliva and bloodstream. Differently, peptides with various degrees of glycosylation corresponding to the C-terminus of SMR1 protein have been isolated and characterized from the saliva of male rat. They are generated by limited cleavage at the monobasic Arg70 site (in collaboration withNG Seidah, IRCM, Montreal). In addition to the undeca-, hexa-, and pentapeptides matured from SMR1 precursor by selective cleavage at pairs of basic amino acid residues, an heptapeptide named SGP-T and, probably generated from the C-terminus of SMR1. has been identified from rat SMG (32).

Taken together, all these findings provide convincing evidence that the androgen-dependent SMR1 protein, mainly produced by the rat SMG, is a novel prohormone. It is a precursor of mature peptides in a way similar to the preproopiomelanocortin hormone precursor which is mainly produced by the pituitary gland and generates distinct peptides involved in variety of local and peripheral biological events (33).

\section{REGULATION OF SH/IR1 (VCSA1) GEME EXPRESSION}

\section{- Differential accumulation of $\mathrm{mRNA}^{\mathrm{SMR1}}$}

TheSMRl transcripts are about 1000 fold more abundant in the SMG of male than female rats. Androgens were shown to be involved in this regulation, and castration results in the decrease of $\mathrm{mRNA}^{\mathrm{SMRI}}$ accumulation in the rat SMG, while injection of androgens to castrated males or to females induces mRNA ${ }^{\text {SMR1 }}$ accumulation at a level similarto that of intact males (10). Whether the effect of androgens on VCSA1 expression is direct or not, is at pesent not known. However, nuclear run on experiments demonstrate that at least part of this regulation occurs at a transcriptional level (Rosinski-Chupin, unpublished data) and potential androgen-responsive elements are present in the
VCSA1 sequence (11). While the other previously studied androgen-regulated genes of the SMG are expressed in the cells of the granular convoluted tubules, the SMR1 transcripts were only detected by in situ hybridization in the acinar cells of rat SMG (25). As expected, SMR1 transcripts are present in high concentrations in all the acinar cells of male glands. In the fema les only some cells appear positive.

\section{- Differential biosynthesis of SMR1 mature peptides}

The quantitative profile of tissue expression and secretion of peptides maturated from SMR1 precursor was earned out using a radioimmunoassay (RIA) specific for the $\mathrm{C}$-terminus of the three peptides, including the undeca-, hexa-, and pentapeptides, and a combination of high performance liquid chromato-graphy (HPLC) analysis.

The biosynthesis of the peptides generated from SMR 1 by cleavage at pairs of arginine residues is subject to distinct regulatory pathways depending on (Tjthe organ, with selective accumulation in SMG and prostate, (ii) the sex, with predominant accumulation in the male gland, and (in) the age of the rat with delectability from 6 week-old SMG. The highest accumulation ofSMRl mature peptides is in the SMG of adult male rat from ten weeks onwards (maximum value represents about 10 mg peptides per gland) (31). In accordance with the demonstration of androgen-induced expression of $\mathrm{mRNA}^{\mathrm{SMRI}}$, the SMG accumulation of SMR1 mature peptides appears to be also dependent on the integrity of the hypothalamic-pituitary-gonad axis. In-, deed, in 4 week-old hypophysectomized or gonadectomized male rats, the accumulation of mature peptides measured 2 or 5 weeks later, respectively, is highly reduced, e.g. close to one or two orders of magnitude less abundant than in the SMG of sham-operated animals (Fig. 3). For various reasons inherent to surgical procedures and its physiological consequences, the pituitary gland ablation is less effective than gonad's, in term of circulating testosterone levels as well as of SMG accumulating SMR1 peptide levels. Therefore, a close relationships between the levels of glandular SMR1 peptide accumulation and that of plasma testosterone concentration was able to be established. Otherwise, female SMG is characterized by low levels of mature peptides, in all experimental conditions, e.g. close to two orders of magnitude less abundant than in male SMG and, by wide variability among individuals.

\section{REGULATION OF SMR1 -HEXAPEPTIDE AND SMR1 - PENTAPEPTIDE SECRETION}

\section{- Differential exocrine secretion}

The exocrine secretion of polypeptides of rodent SMG origin is known to be regulated by the autonomic nervous system, main- * ly through a process mediated by a- or $\mathrm{p}$-adrenergic receptors 


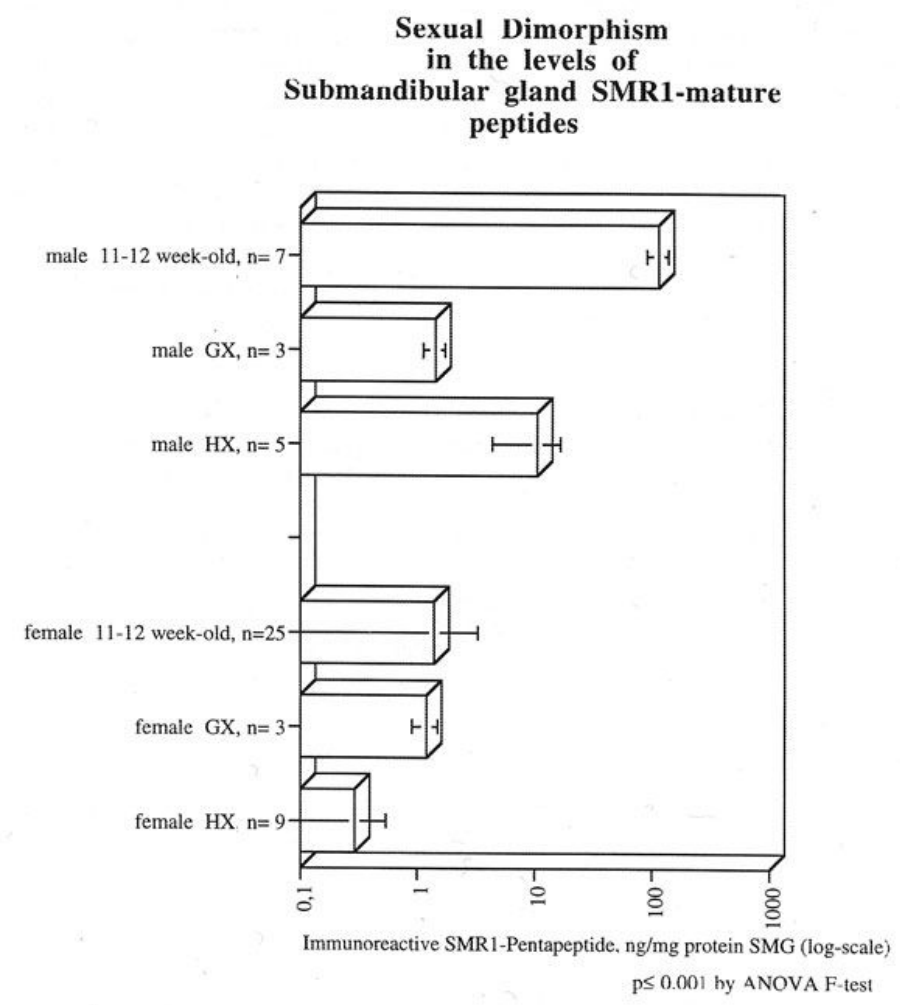

Figure 3. Levels ofSMRl mature peptides in the adult male and female SMG, following pituitary and gonad gland ablations. Results are expressed as ng SMR1 mature peptides/mg total proteins and are the mean - SD of independent experiments. See text and references for experimentaldetails. GX, gonadectomy: HX, hypophysectomy. Glandablation experiments and the effects on the SMG accumulation of tnRNA $\mathrm{TM}^{\mathrm{T}^{\prime \prime} *^{\prime}}$ as well as SMRl-peptides, all suggest that the biosynthesis ofSMRl precursor and derived peptides is under sex hormone control, in particular androgens.

(34,35). The SMR1 mature undeca-, hexa- and pentapeptides which accumulate in the adult male rat SMG are released into the saliva of anaesthetized animals in basal condition and in response to pharmacological stimuli. Release of these peptides is drastically increased in the male rat saliva after the administration of a-adrenergic agonist, epinephrine, or padrenergic agonist, isoproterenol (maximum value represents 1 -2 $\mathrm{mg}$ peptides over the SOminpostinjection period of saliva collection) (31,36) (Fig. 4). This result is in accordance with mRNA $^{\text {SMRI }}$ distribution in the acinar cells of SMG (25). Indeed, protein secretions from SMG acini have been described to be essentially under adrenergic control (37).

\section{- Differential endocrine secretion and characteriza tion of circulating SMRT maturation peptides}

A combination of HPLC analysis and RIA detection, performed on methanol-extracted rat plasma, demonstrate that SMR1 peptides, aside from their exocrine transport, are also carried by the circulation of adult male rats (31). Thus, from adrenergic receptor agonist-treated male rats, the circulating SMR1 peptides correspond primarily to the SMR1 -pentapeptide (56 $\%)$, the rest corresponds to the SMRl-hexapeptide (35\%) and at lesser extent to the SMR1 -undecapeptide (10 \%) (Fig. 5). Interestingly, the plasma SMR1 -pentapeptide can be immunoquan- 
tified by RIA only if blood sampling and extraction steps are carried out in the presence of strong metal chelating agent (EDTA, DTP A), which is not required for the plasma SMR1hexapeptide detection (43). This observation indicates that the acid- and methanol- undissociating pentapeptide complex involves a plasma molecular species including a mineral ion element. Otherwise, as is generally observed for major hormones, the final maturation product of SMR1, SMR1 QHNPR, circulates bound to plasma molecules most likely to facilitate its transport to targets and/or regulate its uptake.

\section{- Sympathetic control of endocrine secretion}

From anaesthetized adult male rats, (3- or ap-adrenoceptormediated exocrine secretion of mature peptides results in their simultaneous endocrine secretion, demonstrating the adrenergic requirement for the local as well as the systemic secretion of SMRl mature peptides. This systemic secretory adrenergic response is not observed in rats in which the SMG ducts and blood vessels are ligated, demonstrating that these circulating peptides originate mainly from the SMG (Fig. 6A). Moreover, since blood peptide levels are maximal within 10 to $30 \mathrm{~min}$ after pharmacological induction, it is likely that secretion occurs directly from the salivary compartment into the blood circu lation rather than through absorption by the gastrointestinal tract (Fig. 6B) (31,36).

\section{- Differential release in conscious and anesthetized rats}

As established for endocrine hormone secretion, the SMG' responsiveness to secretagogue is modified under anesthetized conditions. Indeed, under basal, as well as adrenergic-induced conditions, the circulating level of SMR1 mature pep-

\section{In vivo, salivary secretion \\ of SMR1 mature peptides in adult male rats}

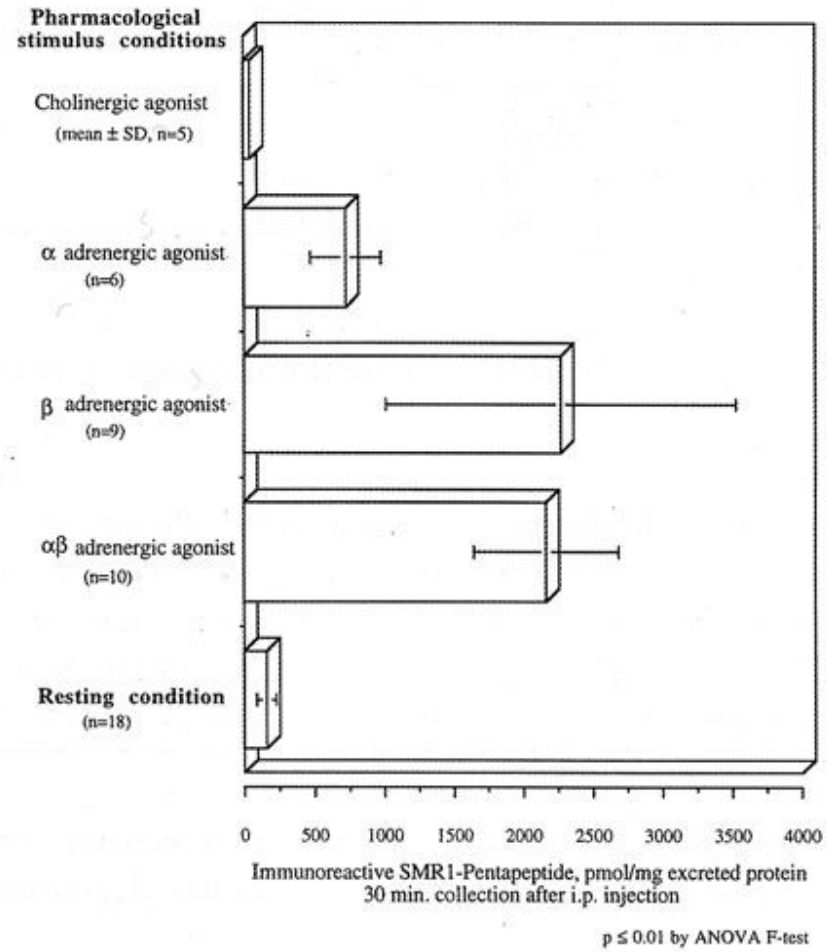

Figure 4. Release of SMRl mature peptides from SMG into the saliva of adult male rats, under basal and aittonomic receptor agonist-induced conditions. Results are expressed as pmol SMRl-pentapeptide/mg total excreted protein over the 30 min. postinjection period of saliva collection, and are the m ean - SD of independent experiments. See text and references for experimental details. The cholinergic agonist pilocarpine was injected i.p. at $1 \mathrm{mg} / \mathrm{kg}$, thea-adrenergic agonist norepinephrine at $2.5 \mathrm{mg} /$ $\mathrm{kg}$, the /3-adrenergic agonist isoproterenol at $0.5 \mathrm{mg} / \mathrm{kg}$, and lhea/3-adrenei-gic agonist epinephrine at $2.5 \mathrm{mg} / \mathrm{kg}$. The salivary secretion of the SMRl mature peptides from SMG is stimulated both by a-and /3-adrenergic mechanisms, however the /3adrenergic response is more intense. 
tides in adult male rats is 5 to 10 times more elevated in conscious rather than analgesic, hypnotic and/or myorelaxant anesthetized animals: $] .9^{ \pm} 0.2 \mathrm{ng} / \mathrm{mlversjjs} 0.4^{ \pm} 0.1 \mathrm{ng} / \mathrm{ml}$ under basal conditions and ]2.5-5Ang/mversus $1.2^{ \pm} 0.2 \mathrm{ng} / \mathrm{ml}$ under adrenergic stimulations, respectively (Fig. 7).

This is why we also explored in conscious rats the ability of SMG to respond to pharmacological and/or to endogenous autonomic receptor activation and thereafter, to establish the physiological range of circulating SMR1 peptides in vivo. The amounts of SMR1 peptides produced by SMG is sexdependent and apparently androgen-related. Strikingly, in conscious rats, un- der basal conditions, the sexual dimorphism of circulating SMR1 peptides is not as significant: $1.9^{ \pm} 0.2 \mathrm{ng} / \mathrm{ml}$ for males vs $0.7^{ \pm} 0.5$ $\mathrm{ng} / \mathrm{ml}$ for females, although the male SMG still produces more than 100 times greater quantity of the peptides compared to female SMG (31). Besides, the pharmacological studies in awake adult males confirm the differential sensitivity of the glandular response to adrenergic receptor agonists in the SMR1 peptide systemic secretion. For instance, both the phenylephrine aadrenergic agonist plus the isoproterenol $\mid 3$-adrenergic agonist are required to induce extended SMR1 peptide secretory response (Fig. 7). Furthermore the endogenous adrenergic secretory response to acute ether-stress results in a SMR1 peptide

\section{In vivo systemic secretion \\ of SMR1 mature peptides \\ HPLC analysis}

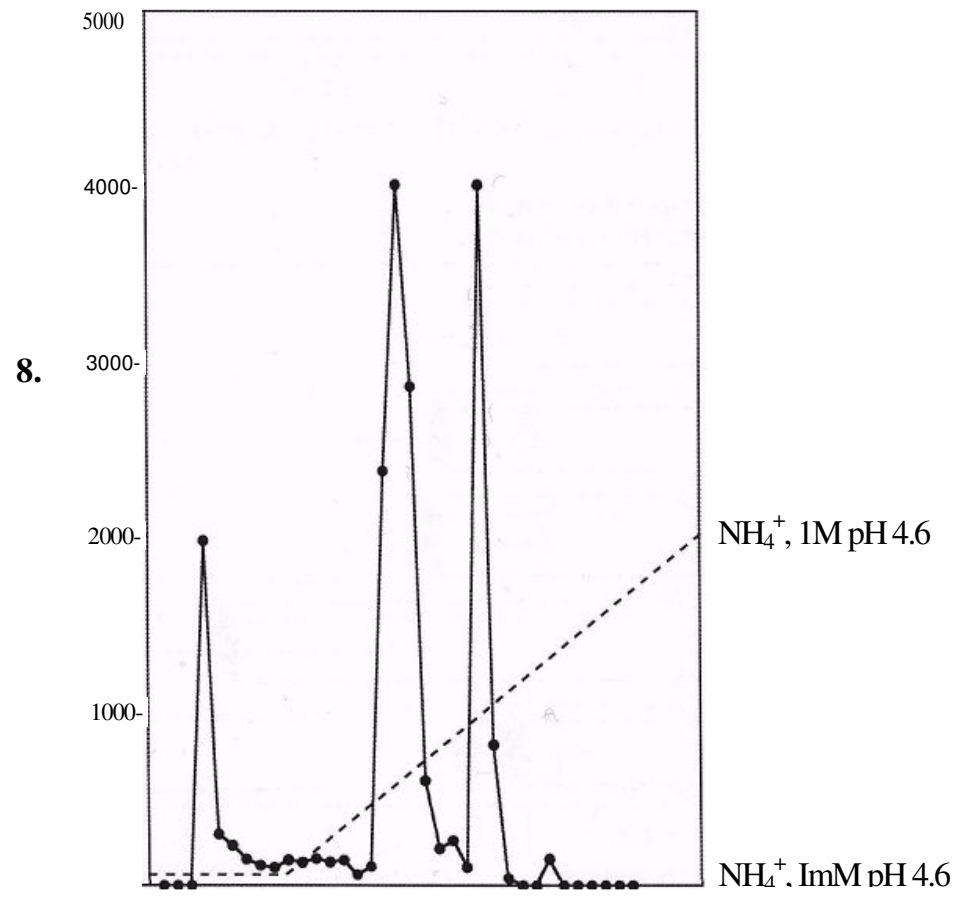

Fraction, $\mathrm{ml} / \mathrm{min}$

Figure 5. Cationic exchange high performance liquid chromatography of methanol-exti'acted plasma obtained from a-adrenergic agonist-treated adult male rats. The representative profile is obtained on HEMA-IEC-BIO-WOO carboxymethyl column, with one step, $30 \mathrm{~min}$ linear gradient of 1-1000 mM ammonium acetate, $\mathrm{pH} 4.6$, at I ml/min flow rate. Fractions are tested after lyophilisationfor their immunoreactive SMRl-pentapeptide by a specific RIA (31). From pharmacological-treated male rats, 56 $21 \%, n=6$ of the plasma SMR1-peptide levels corresponds to free pentapeptide (eluted at 17-19 min), the rest corresponds to hexapeptide (24-25 $\mathrm{min}$ ) and undecapeptide $(29 \mathrm{~min})$. Fraction eluted at 4 min represents the undissociated part of the acid-and methanol-soluble circulating pentapeptide complex. 


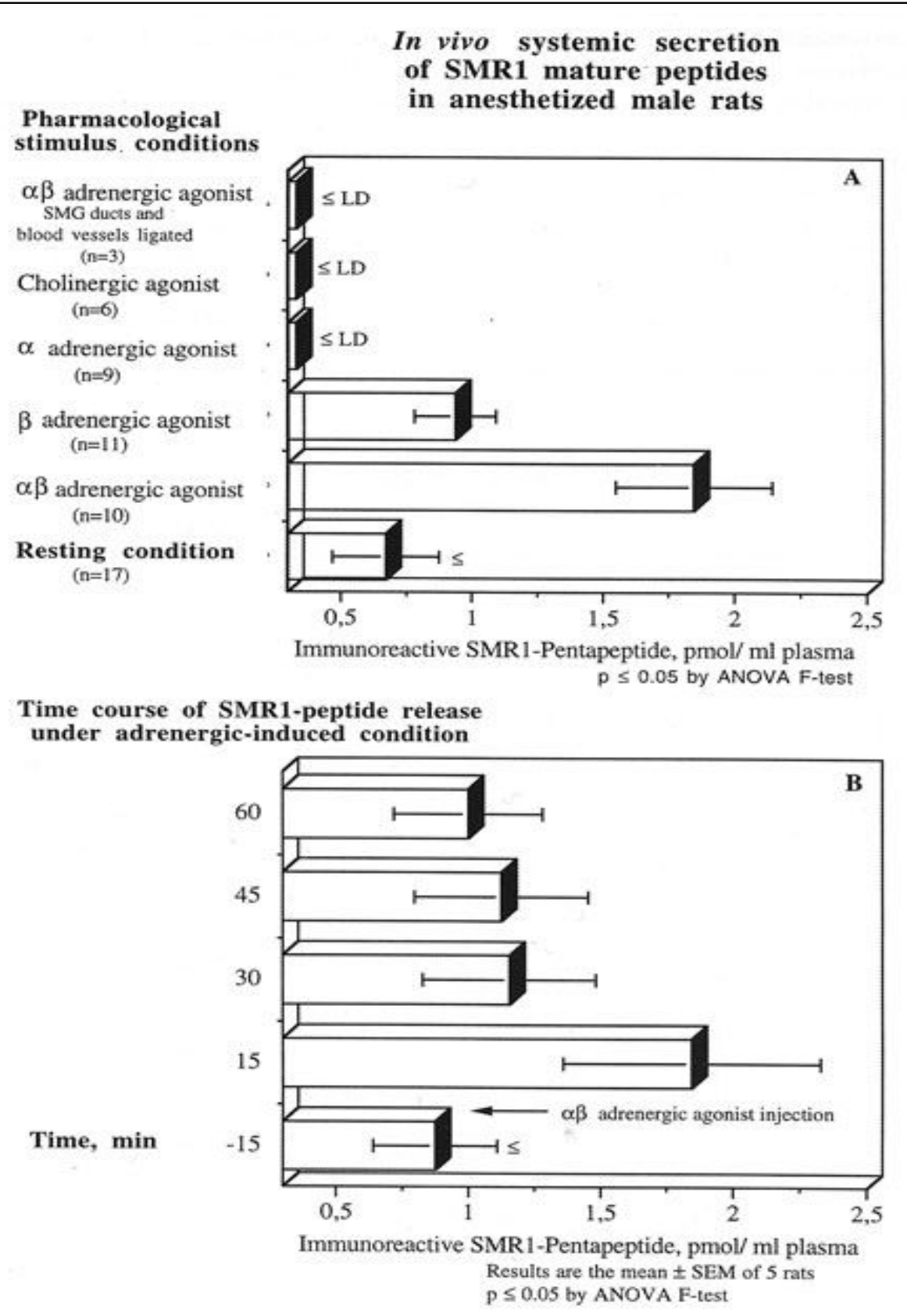

Figure 6. The pharmacological-induced (A) and time-course (B) secretory response of SMR1 mature peptides into the bloodstream of anaesthetized adult male rats. Results are expressed as pmol SMRl-pentapeptide/ml plasma withdrawn 15 min before and 15 to $60 \mathrm{~min}(B)$ or $15 \mathrm{~min}(A)$ after secretogogue administration, and are the mean-SD of independent experiments. See text and references for experimental details. The cholinergic agonist pilocarpine was injected i.p. at $1 \mathrm{mg} / \mathrm{kg}$,theaadrenergic agonist norepinephrine at $2.5 \mathrm{mg} . \mathrm{kg}$, the J3-adrenergic agonist isoproterenol at $0.5 \mathrm{mg} / \mathrm{kg}$, and the afl-adrenergic agonist epinephrine at $2.5 \mathrm{mg} / \mathrm{kg}$. The rapid systemic secretion of the SMR1 mature peptides from SMG is at least under the positive control exerted by the sympathetic nervous system. Otherwises, the process is abolished from male rats in which the SMG ducts and blood vessels were surgically ligated. 
circulating levels of $7.0 \pm 4.1 \mathrm{ng} / \mathrm{ml}(43)$.

Integrating HPLC analysis and these studies, one can establish the physiological range of circulating SMRl-pentapeptide to 1-7 $\mathrm{ng} / \mathrm{ml}$ in adult male rats. From these observations, one canpose an intriguing question about the physiological control and route by which the SMRI peptides enter the circulation from the site of synthesis in the SMG acini (25).

\section{- How is this differential secretion orchestrated?}

One possibility is that SMRl peptide systemic secretion is controlled by morphologically and functionally distinct regions of the SMG, under separate secretory mechanisms and under separate autonomic nerve control. Differences in the involvement of a- and (3-adrenergic receptor induction in protein secretion by the acinar and granular duct cells in the rat SMG are well documented (34,3 5,3 7).

First step, from the site of synthesis to the ductal lumen: The evoked "b-type" response on the SM R1 peptide release into the SMG excretory ducts is in accordance with the cellular site of synthesis and assumes that the exocrine secretory step from the
SMRl -expressing acinar cells into the lumen of the duct is under the predominant control of (3-adrenoceptors. Second step, from the ductal lumen to the bloodstream: injection of radiolabeled SMR1 -pentapeptide into the main SMG excretory duct of male rats results in its rapid appearance in the bloodstream occurring even under basal conditions and being augmented under adrenergic-induced conditions. Furthermore, using recently characterized mouse SMG-derived ductal epithelial cell lines (3 8), we investigated the ability of these polarized cells, when grown as tight monolayers on permeable filter supports, to transcellular transport SMRl-pentapeptide. When $2 \mathrm{ng}$ radiolabeled peptide is added to the apical medium, $10 \%$ of the initially surfaceexposed peptide is recovered in the basolateral medium after six hours. Transcytosis of SMRl pentapeptide is time-dependent with a half time close to $120 \mathrm{~min}$, and in the apical-to-basolateral direction is equal efficient with $\mathrm{EGF}(8 \%$ for 2 ngEGF).

This demonstrates that a fraction of SMRl -pentapeptide is able to be transported in the apical-to-basolateral direction across the SMG ductal cell and subsequently released into the basolateral compartment. However, in contrast to transcytosis of EGF that occurs preferentially in the basolateral-to-apical direction, the transcytosis of SMRl-pentapeptide occurs equally well in

\section{In vivo systemic secretion of SMR1 mature peptides}

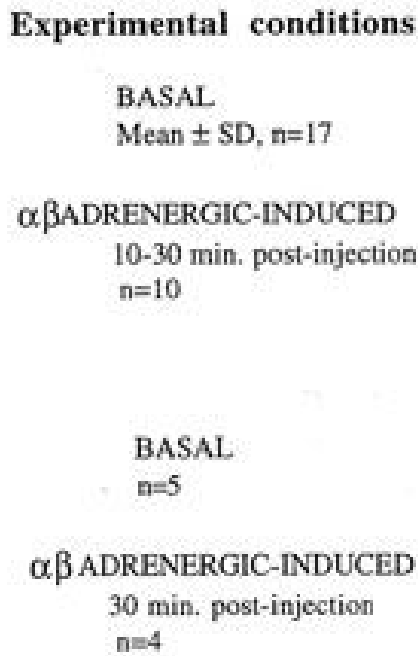

Experimental conditions $n=4$

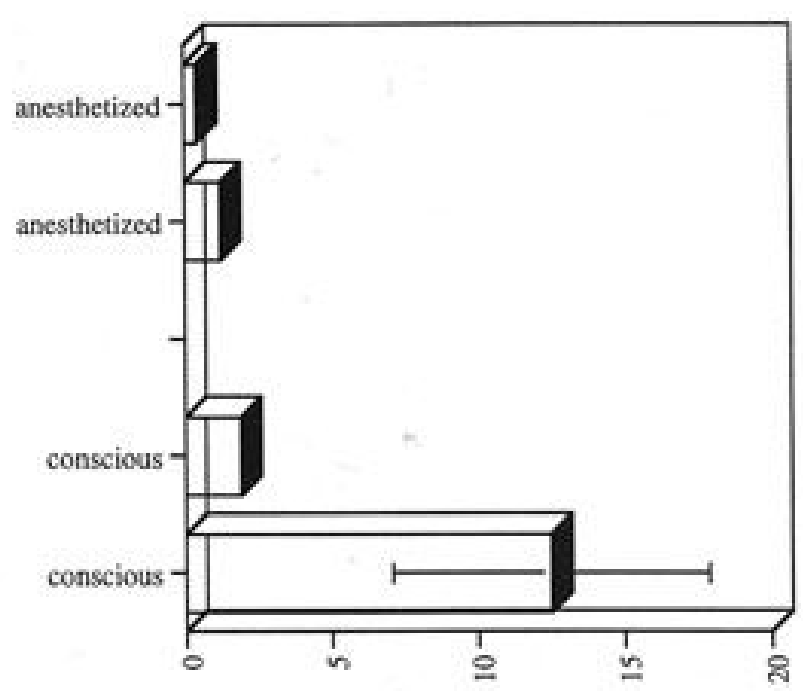

Immunoreactive SMR1-Pentapeptide, ng/ml plasma

Figure 7. Basal and afi-adrenomimelic agonist-induced systemic secretory response of SMRl-peptides Jrom anesthetized 01 conscious adult male rats. Results are expressed as ngSMR I-pentapeptide/ml plasma withdrawn 30 min after pharmacologica induction, and are the mean-SD of independent experiments. See text and references for experimental details. The a-adrenergic agonist phenylephrine plus the /3-adrenergic agonist isoproterenol were injected i.p at $4 \mathrm{ing} / \mathrm{kg}$ and at I mg/kg, respectively From conscious as from anesthetized male rats, the systemic secretion of SMRl mature peptides is under the positive contra exerted through adrenergic receptor activation, however the secretory responsiveness is affected by hypnotic, analgesic and, or mvorelaxant anesthetics. 
both in the apical-to-basolateral and basolateral-to-apical directions. As a control, in the same experiments, no transport is detected for phenol red, a noninvasive probe which assess the development of epithelial confluence and tight junction formation (Laoide et al, manuscript in preparation).

While the exact route of transcellular traffic across epithelial ductal cells is not yet known, all these observations indicate that a fraction of the SMR1 mature pentapeptide which is being routed into the lumen of the duct, could be transported across the ductal cells, where it can access fenestrated capillaries underlying the duct and subsequently reach the circulation. However, further experiments are required in order to identify the mechanisms of: (i) transcytotic pathway across the polarized ductal cells, and (ii) hormonal and neural controls which in vivo direct the systemic secretion under specific physiological behavioral and environmental conditions.

\section{- $\quad$ SMR1 peptide systemic secretion in response to en vironmental stress conditions}

The exposure of rats to saturated ether vapor for two minutes is used as a acute stress-response model to study the endogenous secretion of factors in an integrated physiological context. For example, its effect on endogenous adrenalin and adrenocorticotropin secretion, two of the major mediators of the stress response, is well known $(39,40)$. .... • •

In adult male rats, the systemic secretion of the SMR1 mature peptides is rapidly stimulated in response to exposure of ether fumes $(2 \mathrm{~min})$, while there is no detectable extended release in response to a long exposure to stressful ambient temperatures $\left(120 \mathrm{~min}\right.$ at $4^{\circ} \mathrm{C}$ or $37^{\circ} \mathrm{C}$ ). The response to acute stress stimuli in conscious male rats results in increase of the same order of magnitude in circulating levels of SMR1 peptide as those obtained under pharmacological adrenergic-induced conditions: $7.0 * 4.1 \mathrm{ng} / \mathrm{ml}$ versus $12^{\wedge} * 5.4 \mathrm{ng} / \mathrm{ml}$ compared to basal circulating values, $1.9^{ \pm} 0.2 \mathrm{ng} / \mathrm{ml}$. This suggests that the circulating SMR1 peptide surge is probably initiated and mediated by an endogenous adrenergic secretory response to acute stress.

Complementary to the above experiments it will be necessary to determine whether other types of environmental stress result in alterations in circulating SMR1 peptides, e.g. exposure to coldwater, to harmful photic or acoustic conditions as well as physical restraint or isolation. All these stressors are well known to activate the neuroendocrine hypothalamic-pituitary-adrenal axis $(39,41,42)$. Furthermore, determining the circulating levels of SMR1 peptides, under behavioral stressful conditions such as fighting among males, might give rise to data of physiological significance. Another important aspect of the relationships between the CST-SMG axis and the response to different physiological situations (32) will be to investigate, the effects of he- modynamic (haemorrhage), hormonal (insulin-induced hypoglycemia), and thermoregulatory changes (inflammatory pyretic agent-induced fever) on circulating SMR1 peptide levels.

\section{PERIPHERAL TARGETS FOR CIRCULATING SMR1- PENTAPEP-TIDE}

- As is basically observed for major hormones of the endocrine system, SMR1-pentapeptide is selectively matured from the precursor by cleavage at pairs of basic residues, and is differentially accumulated and systemically released under multifactorial neuroendocrine control. What is the fate of the final secretory maturation product of SMRI (SMR1-QHNPR) when it arrives in the bloodstream from the saliva? Knowing its pharmacodistribution characteristics in vivo should allow one to deduce the sites of action, a crucial step in the determination of the role of this specific SMG factor.

\section{- $\quad$ Blood SMRI-pentapeptide distribution and elimina tion}

As supported by the pharmacokinetic analysis, when discharged into the blood compartment of male rats, the SMG-derived pentapeptide is immediately and widely distributed in a volume of $35-40 \mathrm{ml} / 100 \mathrm{~g}$ body weight, which is similar to the total extracellular fluid volume and is in accordance with the hydrophilic property of the peptide. Thereafter, the peptide disappears from the blood compartment with a distribution half life of close to six minutes, resulting from a rapid distribution to target tissues and/or metabolism and excretion. The elimination stages occur through metabolism in the circulation medium, predominantly from the pentapeptide $\mathrm{N}$-terminus, and through glomerular filtration (43).

\section{- Targettissuedistribution}

By the use of in vivo labeling methods coupled to quantitativeradio image analysis of whole rat body sections, peripheral targets of the final secretory maturation product of SMR1 have been identified. The circulating pentapeptide is taken up selectively by the renal outer medulla, bone (in particular the peri-osteal surface and inner bone), dental tissue and glandular gastric mucosa, as well as pancreatic and submandibular lobules. The characteristics of tissue uptake allow specific binding sites for SMRl-pentapeptide to be identified in vivo. As a matter of fact the binding is rapid (at least $90 \mathrm{sec}$ after bloodstream accession) and stable, is tissue-specific and saturable (dis-placeable in presence of an excess of peptide). Furthermore, the peptide hormone binds to tissue receptor sites at adult male rat physiological circulating concentrations. Direct evidence shows that pentapeptide binding sites are localized in selective portions of the rat nephron, within the epitheli- 
al cells of proximal tubule segments which play a major role in the hormonally regulated hydromineral reabsorption and secretion processes. The existence of pentapeptide binding sites localized within tubular channel processes of trabecular bone, alveolar bone and dentinal layers, which are involved in the deposition and resorption of mineral ions, gives additional evidence that the SMR 1-pentapeptide might be a novel SMG hormonal modulator of mineral balance. Otherwise, pancreatic acinar cells as well as salivary glands are still sites of ion handling and regulation. The differential pentapeptide uptake by these tissues supports the hypothesis of a regulatory role of SMR1 derived peptide in the systemic control of mineral transport. Besides, when one considers that among the targets for the pentapeptide, the gastric chief cells, pancreatic acinar cells and submandibular tissues have a functional role in early digestive processes, the SMR1 peptide maybe also recognized as a potential secretagogue for digestive enzymes (43).

The SMR 1-derived undecapeptide and hexapeptide also reach the bloodstream of adult male rats. Undecapeptide has a pharmacodistribution pattern similar to that of the pentapeptide, in fact, this peptide is efficiently metabolized to the pentapeptide in the circulation medium. However, the time course of tissue distribution of SMR 1-hexapeptide remains to be determined. Overall, from these studies realized under physiological circulating concentration of pentapeptide, it is important to stress the biological relevance of the peripheral pentapeptide uptake in vivo which is consistent with the presence of functional cellular receptor sites.

Based on the available evidence of the circulating SMR1 pentapeptide selective, rapid, stable and saturable uptake by target tissues due to the presence of specific binding sites, one can propose that the androgen-regulated SMR 1-derived peptides might principally participate as an endocrine factor in maintaining systemic mineral homeostasis between the skeletal, the dental and the renal mineral transports (Fig. 8).

\section{CONCLUSION}

- $\quad$ Evidence of physiological significance demonstrate the existence of a CST-SMG neuroendocrine axis involved in maintaining the rat interior milieu (44). SMR1 precursor, and its derived peptides selectively activated in a fashion analogous to that ofpolypeptide hormones of more classical neuroendocrine system, is at present the only SMG specific components of the CST-SMG axis. The gene encoding SMR 1 protein, is a member of the VCS family and it is submitted to transcriptional activation by gonodal steroids, in particular androgens. Thus, its expres sion products which are localized in acinar cells of rat SMG display a high level of sexual dimorphism. As for major hor mones of the endocrine system, the systemic release of the peptides matured from SMR1 precursor, is under the control of sympathetic nervous system in particular through a process mediated by a(3-adrenergic receptors. The physiological range of circulating SMR1 final mature pentapeptide (QHNPR) in conscious adult male rats was established to be $1-7 \mathrm{ng} / \mathrm{ml}$ plasma depending on the state of activation of adrenoreceptors controlling the secretory responsiveness of the SMG. In turn, the circulating SMR1 pentapeptide originating from the SMG, is selectively taken up by peripheral targets through specific binding sites. Hence, localization of primary targets for SMR 1 pentapeptide suggests a physiological involvement of the circulating androgen-dependent peptide in modulating systemic mineral ion balance in vivo.

The androgen-regulated hormone, the SMR1 pentapeptide is released into the bloodstream in basal condition and in response to adrenomimetic agonist administration orto acute stress stimulus. The physiological means and control mechanisms by which the peptide leaves the acinar cells and finds its way to the circulation in vivo has not been yet identified. However, integrating in vitro and in vivo studies, one possible way that this may occur is via differential distribution mechanisms within local and peripheral fluid from the site of synthesis in acini to the effluent blood vessels of the SMG, as follows: $(i)$ by a regulated synthesis and secretory pathway which occurs predominantly through androgen and a-adrenergic receptors respectively, the peptide accesses salivary fluid, and (ii) thereby following a transcytotic pathway across epithelial ductal cells which is presumably effective through (3-adrenergic receptors, the peptide can access adjacent capillaries and therefore reach the circulation. Finally, circulating SMR1 pentapeptide is specifically taken up by peripheral tissues involved in mineral balance. It is therefore apparent that SMR1 peptides, the products elaborated by the SMG, could exert hormonal actions on distal cellular targets to enable coordination between the different components of the organismic response.

This endocrine loop between the sites ofproduction of SMR1related pentapeptide and sites of uptake and potential action can constitute a "long-loop" adjustment system adapted to the species-specific environmental, behavioral and physiological characteristics. For example, associated with male rat-specific behavioral characteristics, e.g. fighting, the SMR 1 -derived pentapeptide may be a SMG paracrine and endocrine factor which under stressful circumstances is acutely secreted to check and balance the local and systemic mineral homeostatic responses to stress. In relation with female rat-specific physiological characteristics this dynamic regulatory loop may match mineral intake and accretion against mineral losses, during stages that requires particular adjustments such as pregnancy and lactation (45). Finally, in one such integrative control mechanism, the SMG might take a remarkable place as a glandular system, participating in the stability of internal environment, which is remarkably also open to the variability of external environment. 


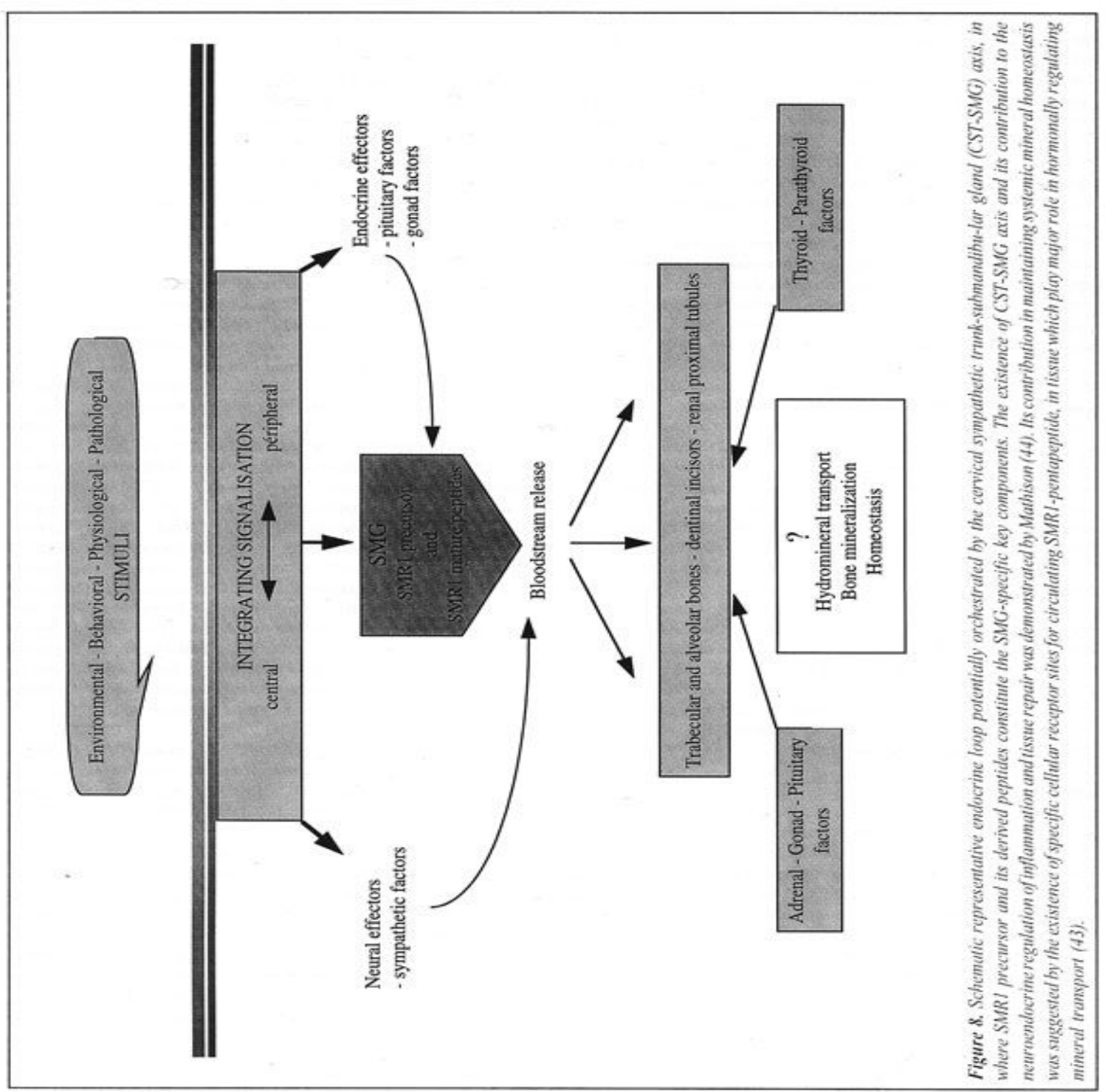

Biomed Rer 9. 1998 


\section{ACKNOWLEDGMENTS}

- $\quad$ These studies were supported by grants from the InstitutPasteurandtheDRET(contract93/l 13 DRET). WethankL. Boutout for secretarial assistance and V. Hermitte for technical assistance. We especially thank DrG. Langley for critical read ing of the manuscript.

\section{REFERENCES}

1. Barka T. Biologically active polypeptides in submandibular glands. J Histochem Cytochem 19\&Q; 28:836-859.

2. Mori M, Takai Y, Kunikata M. Review: biologically active peptides in the submandibular gland - role of the granular convoluted tubule. Acta Histochem Cytochem 1992; 25: 325-341.

3. Wilson CM, Cherry M, Taylor B A, Wilson JD. Genetic and endocrine control of renin activity in the submaxillary gland of the mouse. Biochem Genetics 1981; 509-523.

4. LadenheimRG,SeidahNG, Rougeon F.N-linkedglycosylation affects the processing of mouse submaxillary gland prorenin in transfected AtT20 cells. Ew JBiochem 1991; 198:535-540.

5. Bing J, Poulsen K. In mice aggressive behaviour provokes vast increase in plasma renin concentration, causing only slight, if any, increase in blood pressure. Acta Physio/Scand 1979; 105:64-72.

6. Panthier JJ, Rougeon F. Kidney and submaxillary gland renins are encoded by two non-allelic genes in Swiss mice. £MBO/1983;2:675-678.

7. Mullins HJ, Burt DW, Windass JD, Me Turk P, George H, BrammarWJ. Molecular cloning of two distinct renin genes from the DBA/2 mouse. £M5(9J 1982; 1:1461-1466.

8. Holm I, Olio R, Panthier JJ, Rougeon F. Evolution of aspartyl proteases by gene duplication: the mouse renin gene is organized in two homologous clusters of four exons. $E M B O$ J1984; 3:557-562.

9. Ekker M, Tronik D, Rougeon F. Extrarenal transcription of the renin genes in multiple tissues of mice and rats. ProcNatl AcadSciUSA 1989; 86:5155-5158.

10. Rosinski-Chupin I, Tronik D, Rougeon F. High level of accumulation of a $\mathrm{mRN} A$ coding for a precursor-like protein in the submaxillary gland of male rats. ProcNatl AcadSci USA. 1988; 85:8553-8557.

11. Rosinski-Chupin I, Rougeon F. The gene encoding SMR1, a precursor-like polypeptide of the male rat submaxillary gland, has the same organization as the preprothyrotropinreleasing hormone gene. DNA Cell Biol 1990; 9:553-559.

12. Courty Y, Rosinski-Chupin I, Rougeon F. Various transcripts are generated from the VCSA1 gene by alternative splicing and poly(A) processing in the rat submandibular gland. Gene 1995; 162:291-296.

13. Rosinski-Chupin I, Kuramoto T, Courty Y, Rougeon F,
SerikawaT. Assignment of the rat Variable Coding Sequence (VCS) gene family to chromosome 14. Mammalian Genome 1995;6:153-154.

14. Courty Y, Rosinski-Chupin I, Rougeon F. Anew proline-rich protein precursor expressed in the salivary glands of the rat is encoded by a gene homologous to the gene coding for the prohormone-like protein SMR1. JBiolChem 1994;269:520527.

15. Chretien M, Cromlish JA, SeidahNG. Proprotein convertases (PCS): implication in atherosclerosis and Alzheimer disease. Bull Can Soc Biochem Cell Biol 1997; 86-96.

16. Seidah NG, Chretien M. Eukaryotic protein processing: endoproteolysis of precursor proteins. Cur Opin Biotech«o/1997; 8:602-607.

17. Courty Y, Singer M, Rosinski-Chupin I, Rougeon F. Episodic evolution and rapid divergence of members of the rat multigene family encoding the salivary prohonnone-like protein SMR1. MolBiol Evol 1996; 13:758-766.

18. Senorale-Pose M, Rougeon F. The mouse Vcs2 gene is a composite structure which evolved by gene fusion and encodes five distinct salivary mRN A species. Gene 1997; 201: 75-85.

19. Tronik-Le Roux D, Seorale-Pose M, Rougeon F. Three novel SMR1 -related cDN As characterized in the submaxillary gland of mice show extensive evolutionary divergence in the protein coding region. Gene 1994; 142:175-182.

20. Isemura S, Saitoh E. Nucleotide sequence of gene PBI encoding a protein homologous to salivary proline-rich protein P-E.J Biochem (Tokyoj 1997; 121:1025-1030.

21. Isemura $S$, Saitoh E. Molecular cloning and sequence analysis of cDNA coding for the precursor of the human salivary proline-rich peptide P-B. J Biochem 1994; 115:1101 -1106.

22. Isemura S, Saitoh E, Sanada K. Isolation and amino acid sequences of proline-rich peptides of human whole saliva. $J$ Biochem 1979; 86:79-86.

23. Strawich E,GlimcherMJ. Tooth "enamelins" identified mainly as serum proteins. Major "enamelin" is albumin. Eur $J$ Biochem \990; \9\:47-56.

24. Dickinson DP, Thiesse M. cDNA cloning of an abundant human lacrimal gland $\mathrm{mRN}$ A encoding a novel tear protein. CurrEyeRes 1996; 15:377-386.

25. Rosinski-Chupin I, Rougeot C, Courty Y, Rougeon F. Localization of mRNAs of two androgen-dependent proteins, SMR1 and SMR2, by in situ hybridization reveals sexual differences in acinar cells of rat submandibular gland. J Histochem Cytochem 1993; 41:1645-1649.

26. Chretien M, SikstomR, LazureC, MbikayM, BenjannetS, MarcinkiewiczM et al. Expression of the diversity of neural and hormonal peptides via the cleavage of precursor molecules. In: Peptide Hormones as Prohormones: Processing, Biological Activity, Pharmacology. Ellis Horwood, Chi-chester. 1989; 1-25.

27. Farrow SM. Post-transcriptional regulation of hormone 
synthesis, [review]. JEndocrinol 1993; 138:363-368.

28. Farhadi H, Pareek S, Day R, Dong W, Chretien M, Bergeron JJ et al. Prohormone convertases in mouse submandibular gland: co-localization of furin and nerve grolvth factor. $J$ Histochem Cytocheml997;45:795 804.

29. Lindberg I, Mutton JC. Peptide processing proteinases with selectivity for paired basic residues. In: FrickerLD, editor. Peptide Biosynthesis and Processing. Boca Raton, CRC Press, FL. 1991; 141-174.

30. Smeekens SP, Chan SJ, Steiner DF. The biosynthesis and processing of neuroendocrine peptides: identification of proprotein convertases involved in intravesicular processing, [review]. Prog Brain Res 1992; 92:235-246.

31. Rougeot C, Rosinski-Chupin I, Njamkepo E, Rougeon F. Selective processing of submandibular rat 1 protein at dibasic cleavage sites. Salivary and bloodstream secretion products. EitrJBiochem 1994; 219:765-773.

32. Mathison RD, Befus AD, Davison S. A novel submandibular gland peptide protects against endotoxic and anaphylactic shock. $\wedge / 7$ ?. $/$ / $/ 7$ jwo/1997;273:R1017-R1028.

33. Hanneman E, Bunzow J; Salon J, Grandy D, AlbertP. Peptides encoded by the proopiomelanocortin gene.In: Peptide Hormones as Prohormones: Processing, Biological A ctivity, Pharmacology. EllisHorwood,Chichester. 1989; 53-82.

34. Abe K, Dawes C. The effects of electrical and pharmacological stimulation on the types of protein secreted by rat parotid and submandibular ghnds. Arch OralBiol 1978; 23:367-372.

35. GarrettJR, Suleiman AM, Anderson EC, Proctor GB. Secretory responses in granular ducts and acini of submandibular glands in vivo to parasympathetic and sympathetic nerve stimulation in rats. Cell Tiss Res 1991; 264:117-126.

36. Rougeot C, Rougeon F. Processing of SMR1 protein in rat submandibular glands: adrenergic-induced release of GlnHis-Asn-Pro-Arg related peptides in saliva and blood of males. Amino Acids 1993;5:187-188.
37. Anderson LC, Garrett JR, ZhangX, Proctor GB, Shod DK. Differential secretion of proteins by submandibular acini and glandular ducts on graded autonomic nerve stimulations. Jf/riOTb/1 995; 485:503-511.

38. LaoideBM,Courty Y,GastinneI,ThibautC,KellermannO, Rougeon F. Immortalised mouse submandibular epithelial cell lines retain polarised structural and functional properties. JCellSci 1996; 109:2789-2800.

39. Rougeot C, Tiberghein C, Minary P, Dray F. Basal and PAF-, interleukin 1-, ether stress-induced hypothalamic pituitary adrenal secretion of conscious rat: modulation by PAF antagonists. JLipidMediat 1991; 4: 45-59.

40. Van Herck H, Baumans V, de Boer SF, van derGugten J, van Woerkom AB, Beynen AC. Endocrine stress response in rats subjected to singular orbital puncture while under diethyl-ether anaesthesia. LabAnim 1991; 25:325-329.

41. Terrazzino S, PeregoC, De SimoniMG. Effect of development of habituation to restraint stress on hypothalamic noradrenaline release and adrenocorticotropin secretion. J Neurochem 1995; 65:263-267.

42. WeidenfeldJ,RougeotC,DrayF,FeldmanS.Adrenocortical response following acute neurogenic stimuli is mediated by CRF-41. Newosci Lett 1989; 107:189-94.

43. Rougeot C, Vienet R, Cardona L, EeDoledec L, Grognet JM, Rougeon F. Targets for SMRl-pentapeptide suggest a link between the circulating peptide and mineral transport. Am JPhysiol 1997; 273: R1309-R1320.

44. Mathison R., Davison JS, Befus AD. Neuroendocrine regulation of inflammation and tissues repair by submandibular gland factors. Imnnmol Today 1994; 15:527532.

45. BikleDD, Negro-Vilar A. Hormonal regulation of bone mineral metabolism. In: Negro-Vilar A,editor. Endocrine Reviews Monographs. Endocrine Society Press,Bethesda. 1995;4:1-397. 